\title{
Effect of Pre-Existing Immunity to Flaviviruses on Balanced Induction of Neutralizing Antibodies by a Dengue Tetravalent DNA Vaccine in Mice
}

\section{Eiji Konishi ${ }^{1,2, *}$ and Yamato Takizawa ${ }^{1}$}

${ }^{1}$ Division of Infectious Diseases, Department of International Health, Kobe University Graduate School of Health Sciences, 7-10-2 Tomogaoka, Suma-ku, Kobe 654-0412, Japan

${ }^{2}$ Division of Vaccinology, Department of Microbiology and Infectious Diseases, Kobe University Graduate School of Medicine, 7-5-1 Kusunoki-cho, Chuo-ku, Kobe 6500017, Japan

\begin{abstract}
We previously demonstrated that a DNA-based tetravalent dengue vaccine can induce balanced neutralizing antibody responses against all four types of dengue virus (DENV1-4), using naïve mice. On the other hand, individuals receiving the dengue vaccine may have previously been infected with dengue virus (DENV1-4) or vaccinated with other flavivirus vaccines. Thus, we investigated the effect of preimmunity against flaviviruses on immunogenicity of a dengue tetravalent DNA vaccine in mice. Mice preimmunized with DENV2 developed higher antibody levels against DENV2 than other types of dengue virus after the first dengue vaccination. However, the second vaccination provided similar increases (4- to 8-fold) in antibody levels against all types of dengue virus, compared with non-preimmunized mice. Preimmunization with yellow fever or Japanese encephalitis vaccines did not affect the immunogenicity of the tetravalent vaccine, except for slight and occasional increases in antibody levels against certain types of viruses. Thus, the dengue tetravalent DNA vaccine could provide balanced induction of dengue antibody responses even in mice preimmunized with any of the three flaviviruses, at least after the second vaccination.
\end{abstract}

Keywords: Japanese encephalitis; Dengue; Preimmunity; DNA vaccine; Neutralizing antibody

\section{Introduction}

Dengue fever (DF) is a mosquito-borne viral disease widely distributed in most tropical and subtropical regions [7,12]. An estimated 50-100 million human cases occur annually. One of the important features of this disease is the presence of its severe form, dengue hemorrhagic fever (DHF). Approximately 250,000-500,000 cases of DHF have been reported every year. The causative agent of DF and DHF is any of four types of dengue virus (dengue type 1-4 virus; designated DENV1-4), members of the genus Flavivirus in the family Flaviviridae [25]. There are currently no approved vaccines and no specific antiviral drugs available against the dengue virus [14,33].

Epidemiological evidence indicates that once an individual becomes infected with one type of dengue virus they are usually protected from subsequent homotypic infection [30], but disease severity can progress from DF to DHF mostly following heterotypic infection [4,11]. One of the potential mechanisms that leads to this increased disease severity is the presence of non-neutralizing cross-reactive antibodies $[13,19,24]$ and/or cross-reactive memory $\mathrm{T}$ lymphocytes [6] induced by an earlier infection. Therefore, a tetravalent vaccine that can induce immune responses against all four types of dengue virus would be required for developing a safe and effective dengue vaccine [14,33]. It is highly probable that neutralizing antibodies constitute an immunological correlate of protection for dengue, although this is still being debated [15].

Several dengue tetravalent vaccine candidates are currently under development, three of which are in the more advanced stages, namely, traditional attenuation, engineered attenuation and chimerization, which are all being developed via live attenuated vaccine strategies $[14,33]$. Evaluation in clinical trials demonstrated that these vaccine strategies have produced vaccines with high seroconversion rates to all four types of the virus $[3,10,28,29,32,34]$. However, interference in virus replication is a potential problem that may occur when infectious vaccines are combined. This is especially important in the development of dengue tetravalent vaccines, since imbalanced immune responses may cause enhanced disease severity [31]. Therefore, dosage formulations and/or vaccine schedules are considered important to adjust the immunogenicity of the four different live vaccine components $[9,18]$.

DNA vaccines are non-infectious vaccines that incorporate a gene of interest from the infectious agent concerned and they are delivered to the cells of the inoculated host, where the gene is expressed [26]. Since the DNA vaccine strategy does not seem to cause interference in antibody response due to combined immunization, the strategy is considered suitable for developing a dengue tetravalent vaccine. We have constructed and evaluated a dengue tetravalent DNA vaccine consisting of pcDNA3-based plasmids expressing the premembrane (prM) and envelope (E) genes of each of the four types of dengue virus [20]. In a mouse model, this vaccine candidate did not show detectable interference and induced balanced neutralizing antibody responses to all four types of dengue virus.

It is important to consider that individuals residing in endemic areas could have been infected with dengue virus prior to vaccination with a dengue tetravalent vaccine. Similarly, individuals who receive a

*Corresponding author: Eiji Konishi, Department of International Health, Kobe University Graduate School of Health Sciences, Kobe, Japan, 7-10-2 Tomogaoka Suma-ku, Kobe 654-0142, Japan Tel/Fax: +81-78-796-4594; E-mail: ekon@ kobe-u.ac.jp

Received July 30, 2010; Accepted September 25, 2010; Published September 28, 2010

Citation: Konishi E, Takizawa Y (2010) Effect of Pre-Existing Immunity to Flaviviruses on Balanced Induction of Neutralizing Antibodies by a Dengue Tetravalent DNA Vaccine in Mice. J Vaccines Vaccin 1: 102. doi:10.4172/21577560.1000102

Copyright: ( $) 2010$ Konishi E, et al. This is an open-access article distributed under the terms of the Creative Commons Attribution License, which permits unrestricted use, distribution, and reproduction in any medium, provided the original author and source are credited. 
dengue tetravalent vaccine as a traveler's vaccine may have also been vaccinated with Japanese encephalitis (JE) vaccine or yellow fever (YF) vaccine [2]. Since relatively high serological cross-reactivities exist between flaviviruses [23], it is critical to examine if pre-existing immunity to these viral antigens would affect the ability of a dengue tetravalent vaccine to provide a balanced induction of neutralizing antibody responses against the four dengue viruses.

This study assesses the effect of preimmunity to DENV2 infection or JE or YF vaccination on the immunogenicity of our dengue tetravalent DNA vaccine in a mouse model. The results demonstrated that the ability of the tetravalent vaccine to induce balanced antibody responses to all four types of dengue virus was not affected by preimmunization, at least after two vaccinations. We speculate that enhanced induction of broad cross-reactive neutralizing antibodies may assist the balanced induction of dengue antibody responses.

\section{Materials and Methods}

\section{Cells}

Vero and C6/36 cells were cultivated as described previously [35]. Briefly, Vero cells were grown in Eagle's minimum essential medium (MEM) supplemented with $10 \%$ heat-inactivated fetal bovine serum (FBS) and kanamycin $60 \mu \mathrm{g} / \mathrm{ml}$. C6/36 cells were grown under the same conditions except for the addition of nonessential amino acids to the medium. All cells were cultured in a humidified atmosphere containing $5 \% \mathrm{CO}_{2}$ at $37^{\circ} \mathrm{C}$ for Vero cells, or at $28^{\circ} \mathrm{C}$ for C6/36 cells.

\section{Viruses}

Dengue viruses used in this study included the Mochizuki strain of DENV1, the New Guinea C (NGC) strain of DENV2, the H87 strain of DENV3, and the H241 strain of DENV4 [20]. The Nakayama strain of JE virus (JEV) has been described previously [16]. The 17D strain of YF virus (YFV) was provided by Dr. Tomohiko Takasaki of the National Institute of Infectious Diseases (NIID), Japan. Culture fluids harvested from C6/36 cells infected with each of these viruses were used as antigen for the neutralization tests and ELISA. For preimmunization of mice with DENV2, the virus was administered in the form of a $20 \%$ homogenate of infected suckling mouse brains prepared with $10 \%$ normal mouse serum as a stabilizer. For preimmunization with the 17D strain, culture fluids harvested from infected C6/36 cells cultivated with medium containing $1 \%$ normal mouse serum in place of $10 \%$ FBS were used.

\section{Antibodies}

Mouse monoclonal antibodies specific for E proteins included: D1-4G2 (flavivirus group-crossreactive, purchased from American Type Culture Collection, Manassas, VA), D1-I-15C12 (DENV1-reactive, unpublished), D2-II-11H4 (DENV2-reactive; 35) and JE-10B4 [JEVreactive, 22]. Hyperimmune mouse ascitic fluids (HMAFs) were collected from mice repeatedly immunized with DENV1 (Mochizuki), DENV2 (NGC), DENV3 (H87), DENV4 (H241) or JEV (Nakayama) in the form of $10 \%$ homogenates of infected suckling mouse brains [20]. Hyperimmune mouse sera against YFV were collected from mice repeatedly immunized with culture fluid harvested from 17D-infected C6/36 cells cultivated in medium containing $1 \%$ normal mouse serum. Hyperimmune rabbit sera against DENV1-4 were collected from rabbits repeatedly immunized with the purified virion fraction of each dengue virus [20].

\section{Vaccines}

Dengue DNA vaccines were the pcDNA3-based vaccine plasmids encoding the prM and $E$ genes of the DENV1 Mochizuki strain
(pcD1ME), the DENV2 NGC strain (pcD2ME), the DENV3 H87 strain (pcD3ME) and the DENV4 H241 strain (pcD4ME) [20]. A mixture of $10 \mu \mathrm{g}$ of each of these plasmids, pcD1ME, pcD2ME, pcD3ME and pcD4ME (totaling $40 \mu \mathrm{g}$ ), was used as the dengue tetravalent DNA vaccine. All DNAs were purified using a plasmid DNA purification kit (Qiagen, Hilden, Germany). For the YF vaccine, the 17D strain of YFV was used. For the JE vaccine, a mouse brain-derived inactivated JE vaccine (JEVAX) purchased from Mitsubishi Tanabe Pharma (Osaka, Japan) was used.

\section{Mouse experiments}

Groups of four to six 4-week-old male ddY mice (Japan SLC, Shizuoka, Japan) were preimmunized by intraperitoneal (i.p.) inoculation once or twice, with a 2-week interval, with DENV2 antigen (20\% homogenate of infected suckling mouse brains), YFV antigen (culture fluid harvested from infected C6/36 cells) or JEV antigen (JEVAX). Three to four weeks after the preimmunization, mice were vaccinated twice, with a 3- to 4-week interval, with the dengue tetravalent DNA vaccine ( $40 \mu \mathrm{g}$ per mouse) by inoculation into both thighs using a spring-powered needle-free jet injector (ShimaJET; Shimadzu, Kyoto, Japan). The volume of the vaccine solution injected into each thigh was adjusted to $50 \mu \mathrm{l}$ (100 $\mu \mathrm{l}$ per mouse) with phosphate-buffered saline. Mice were retroorbitally bled and sera isolated. Pooled sera were examined for neutralizing antibody titers and individual sera examined for ELISA antibody levels, except for preimmunization experiments with DENV2 in which the pooled sera were used for both neutralization tests and ELISA.

All animal experiments were approved by the Institutional Animal Care and Use Committee (Permission number: P-070101 and P-090615) and conducted according to the Kobe University Animal Experimentation Regulations.

\section{Neutralization test}

Neutralizing antibody titers were determined on Vero cells using a plaque reduction assay as described previously [20]. The antigens used included YFV (17D), JEV (Nakayama), DENV1 (Mochizuki), DENV2 (NGC), DENV3 (H87) and DENV4 (H241). The virus-antibody mixture was incubated in the presence of rabbit complement at a final concentration of $5 \%$. Plaques were visualized by immunostaining using D1-I-15C12, D2-II-11H4, JE-10B4 and D1-4G2 monoclonal antibodies to detect DENV1, DENV2, JEV and other viral antigens, respectively. The neutralizing antibody titer was expressed as the maximum serum dilution yielding a $70 \%$ reduction in plaque number.

\section{ELISA}

A conventional ELISA for measuring antibody levels was performed as described previously [20]. Briefly, microplates were sensitized with hyperimmune rabbit sera against DENV1-4 or JEV and incubated with infected culture fluids containing corresponding viral antigens. For measuring antibodies to YFV, hyperimmune rabbit serum against DENV2 was used to capture YFV antigens, utilizing their cross-reactivity. Sensitized plates were incubated serially with test sera, alkaline phosphatase-conjugated anti-mouse $\operatorname{IgG}$ and $p$-nitrophenyl phosphate. To minimize interplate variations, a constant positive control serum was included in every plate, and the absorbances obtained with the test samples were adjusted with the positive control, which was taken as 1.0 . The adjusted absorbances were expressed as ELISA values. The positive control serum was the pooled serum obtained at the end of the experimental period from mice that had not been preimmunized but had been vaccinated twice with the tetravalent DNA vaccine, unless otherwise specified. The ELISA used to measure $\operatorname{lgG} 1$ and $\operatorname{lgG} 2 \mathrm{a}$ antibodies was the same as 
that described above, except serial 2-fold dilutions of the test sera and alkaline phosphatase-conjugated goat anti-mouse $\operatorname{IgG} 1$ or $\operatorname{IgG} 2 \mathrm{a}$ were used. The ELISA antibody titer was the maximum serum dilution that showed an absorbance value greater than the average, plus three times the standard deviation of the absorbance values obtained with non-immune mouse sera. The $\lg G 2 \mathrm{a} / \lg \mathrm{G} 1$ ratio was calculated from the ELISA antibody titers obtained for $\operatorname{IgG} 1$ and $\operatorname{IgG} 2 \mathrm{a}$ isotypes.

\section{Statistical analysis}

Significant differences in the geometric mean ELISA antibody levels were evaluated using the Student's $t$-test. Probability levels $(P)$ of less than 0.05 were considered significant.

\section{Results}

\section{Cross-neutralization among JEV, YFV and dengue viruses}

Although the neutralization test provides the highest specificity among currently available serological tests to measure flavivirus antibodies, it still detects some cross-reactive antibodies. Since this study aimed to investigate immunogenicity of the dengue tetravalent DNA vaccine in mice with preimmunity to antigens of DENV2, JEV or YFV, we evaluated cross-neutralization among these viruses. For this purpose, hyperimmune mouse ascitic fluids against JEV or each of the DENV1-4 and hyperimmune mouse sera against YFV were used for neutralization tests (Figure 1). Neutralizing antibody titers of 1:640 to 1:5120 were detected against the homologous virus, whereas antibody titers against heterologous viruses were $\leq 1: 40$ and mostly $\leq 1: 10$ in all combinations examined. This result indicated that the cross-neutralization titers were $\leq 1 / 32$ and mostly $<1 / 64$ of the specific titer under these experimental conditions using a $70 \%$ plaque reduction assay.

\section{Effect of pre-existing immunity to DENV2}

To assess an effect of preimmunity to DENV2 on immunogenicity of the dengue tetravalent DNA vaccine, mice were immunized with
DENV2 antigen, in the form of suckling mouse brain homogenates, before vaccination with the DNA vaccine. In the first experiment, groups of six mice were preimmunized with $5.0 \times 10^{7}, 5.0 \times 10^{6}$ or $5.0 \times 10^{5}$ plaque forming units (PFU) of DENV2 antigen. As a control, the same number of mice were inoculated with PBS. At 4 and 7 weeks later, these mice were vaccinated with $40 \mu \mathrm{g}$ of the dengue tetravalent DNA vaccine (10 $\mu \mathrm{g}$ for each type). Nine weeks later (2 weeks after the second vaccination), mice were bled and the pooled sera were examined for neutralizing antibodies (Table 1). Overall, mice preimmunized with higher doses of DENV2 antigen showed higher neutralizing antibody titers. Compared with control mice without preimmunization, preimmunized mice showed a 4- to 16fold increase in neutralizing antibody titers against the homologous antigen (DENV2), and a 1- to 8-fold increase against heterologous antigens (other types).

To further assess the effect of preimmunization with DENV2 antigen, we carried out a second experiment to compare the time course of antibody responses in mice with or without preimmunization. Mice preimmunized with $5.0 \times 10^{7}$ or $5.0 \times 10^{6}$ PFU of DENV2 antigen or inoculated with PBS were vaccinated with $40 \mu \mathrm{g}$ of the dengue tetravalent DNA vaccine twice, with a 4-week interval. Blood samples were collected just before (week 0 ) and every week after the first vaccination, except for week 3 , and pooled sera were examined for neutralizing antibodies and ELISA antibodies (Figure 2).

In mice preimmunized with $5.0 \times 10^{7}$ PFU of DENV2, prevaccination neutralizing antibody titers were $1: 80$ against DENV2, compared with $<1: 10$ against other types. After the first vaccination, antibody titers in preimmunized mice started to increase against DENV2 at week 1 and against other types at week 1 or 2 , whereas non-preimmunized mice did not develop detectable neutralizing antibodies against any of the four types within 2 weeks. Four weeks after the first vaccination, the neutralizing antibody titer against DENV2 in preimmunized mice (1:320) was 32-fold higher than that in



Figure 1: Cross-neutralization among JEV, YFV and DENV1-4. The abscissa indicates virus species used for preparing hyperimmune mouse ascitic fluids (HMAF) or hyperimmune mouse sera (HMS). The ordinate indicates neutralizing antibody titers obtained with HMAF or HMS as determined by the neutralization tests using JEV, YFV and DENV1-4 (indicated in the box).

\begin{tabular}{|c|c|c|c|c|}
\hline \multirow[b]{2}{*}{ Dose of DENV2 } & \multicolumn{4}{|c|}{ Neutralizing antibody titer against: ${ }^{b}$} \\
\hline & & & & \\
\hline antigen $(\mathrm{PFU})^{\mathrm{a}}$ & DENV1 & DENV2 & DENV3 & DENV4 \\
\hline $5.0 \times 10^{7}$ & $1: 160$ & $1: 320$ & $1: 160$ & $1: 160$ \\
\hline $5.0 \times 10^{6}$ & $1: 160$ & $1: 160$ & $1: 80$ & $1: 80$ \\
\hline $5.0 \times 10^{5}$ & $1: 80$ & $1: 80$ & $1: 80$ & $1: 20$ \\
\hline PBS & $1: 40$ & $1: 20$ & $1: 40$ & $1: 20$ \\
\hline
\end{tabular}

aGroups of six 4-week-old male ddY mice were preimmunized with the indicated doses of DENV2 antigen or inoculated with PBS as a control.

${ }^{b}$ Four weeks after the preimmunization, mice were vaccinated with $40 \mu \mathrm{g}$ of the dengue tetravalent DNA vaccine (10 $\mu \mathrm{g}$ for each type) twice, with a 3 -week interval. Two weeks after the second dose of the DNA vaccine, mice were bled and pooled sera were examined for neutralizing antibodies.

Table 1: Neutralizing antibody titers in dengue tetravalent DNA vaccine-vaccinated mice with or without preimmunization with DENV2. 
mice without preimmunization (1:10), while the titers against other types of DENV in preimmunized mice (1:40-1:80) were only 4-fold higher than those in non-preimmunized mice (1:10-1:20). However, two weeks after the second vaccination (week 6), the titer differences between mice with and without preimmunization decreased to 4 - to 8-fold against any type, and developed antibody titers of 1:160 $1: 1280$ and 1:40-1:160, respectively. Mice preimmunized with 5.0 $\times 10^{6}$ PFU of DENV2 showed intermediate time courses between mice without preimmunization and those preimmunized with $5.0 \times$ $10^{7}$ PFU of DENV2. Although ELISA antibody levels were affected by cross-reactivities among DENV1-4 antigens, the time courses were basically consistent with those of neutralizing antibody titers in terms of the difference between mice with and without preimmunization. These results indicated that, although preimmunization with DENV2 increased pre- and postvaccination antibody levels against DENV2 more effectively than those against other types, the differences in antibody levels from those obtained with non-preimmunized mice were similar across the four types (DENV1-4) following the second vaccination.

\section{Effect of pre-existing immunity to JEVAX}

The effect of preimmunization with a commercial JE vaccine (JEVAX) on immunogenicity of the dengue tetravalent DNA vaccine was then investigated. Mice preimmunized with $1 / 2$ or $1 / 10$ of a human dose of JEVAX were vaccinated twice with $40 \mu \mathrm{g}$ of the dengue tetravalent DNA vaccine. Mice were bled at weekly intervals. Pooled sera were examined for neutralizing antibodies, and individual sera were examined for ELISA antibodies (Figure 3). A 1/2 dose of JEVAX induced a higher neutralizing antibody titer against JEV $(1: 80)$ than a $1 / 10$ dose (1:20) prior to vaccination with the dengue tetravalent DNA vaccine.

Different from the results obtained with DENV2-preimmunized mice (Figure 2), mice with or without JEVAX preimmunization showed similar postvaccination neutralizing antibody titers, except for those against DENV1, which were mostly 2-fold and sometimes 4-fold higher in preimmunized than non-preimmunized mice (Figure 3). ELISA antibody levels were significantly higher in preimmunized than non-preimmunized mice against all types of dengue virus before (week 0 ) and/or 1 week after vaccination, due to cross-reactivity between antigens of JEV and DENV1-4. Following the second vaccination (weeks 5 and 6), similar antibody titers were detected between mice with and without preimmunization, against any type

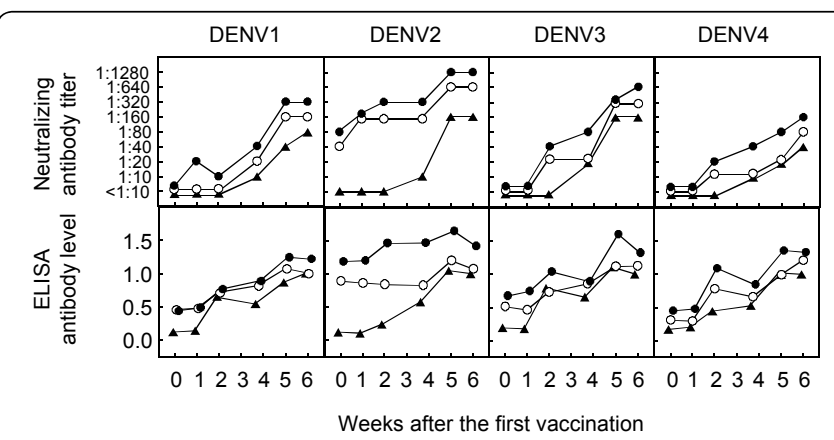

Figure 2: Effect of preimmunity to DENV2 on immunogenicity of the dengue tetravalent DNA vaccine. Groups of four mice were preimmunized with $5.0 \times 10^{7}$ (closed circle) or $5.0 \times 10^{6}$ (open circle) PFU of DENV2 antigen or inoculated with PBS (closed triangle). Four and eight weeks later, these mice were vaccinated with $40 \mu \mathrm{g}$ of the dengue tetravalent DNA vaccine $(10 \mu \mathrm{g}$ for each type). Pooled sera were examined for neutralizing antibody titers (upper panel) and ELISA antibody levels (lower panel) against DENV1-4 (indicated above the upper panel) of dengue virus. These results indicated that preimmunization with JEVAX did not affect the immunogenicity of the dengue tetravalent DNA vaccine under these experimental conditions. As for the neutralizing antibody titers against JEV, they remained unchanged before the second vaccination, after which the titers increased 2- to 4-fold. On the other hand, ELISA antibody levels against JEV constantly increased with time, in mice both with and without preimmunization, due to cross-reactive antibodies detected by ELISA.

\section{Effect of pre-existing immunity to YFV}

To assess the effect of preimmunization with YF vaccine on immunogenicity of the dengue tetravalent DNA vaccine, the YFV 17D strain harvested from culture fluids of infected C6/36 cells was used in place of the commercial YF vaccine. Since one human dose of a commercial attenuated YF vaccine $\left({\mathrm{YF}-V A X^{\circledR}}^{\circledR}\right)$ contains a release titer of $>5.6 \times 10^{4} \mathrm{PFU}$ [27]. We used a virus infective titer of 5.6 $\times 10^{4} \mathrm{PFU}$ as one human dose, however, preliminary experiments indicated that this dose did not induce high neutralizing antibody titers in mice, and so a higher dose $\left(5.6 \times 10^{6} \mathrm{PFU}\right.$ corresponding to 100 human doses) was also used for preimmunization. Preimmunized mice were vaccinated twice with $40 \mu \mathrm{g}$ of the dengue tetravalent DNA vaccine. As shown in (Figure 4), preimmunization with 100 human doses induced a neutralizing antibody titer of 1:160 against YFV, while one human dose induced a titer of $1: 10$. Mice were bled at weekly intervals to examine pooled sera for neutralizing antibody titers and individual sera for ELISA antibody levels.

Similar to the results obtained by preimmunization with JEVAX (Figure 3), mice with or without preimmunization showed similar time courses of antibody titers/levels against each of the dengue virus types, DENV1-4 (Figure 4). Four-fold higher neutralizing antibody titers were detected against DENV1 or DENV2 at a certain time point in mice preimmunized with 100 human doses compared with non-preimmunized mice, while significant differences in ELISA antibody levels were occasionally detected against DENV1 and DENV3 between mice with and without preimmunization following the second vaccination. As for neutralizing antibody titers against YFV, they increased one or two weeks after the first vaccination in preimmunized mice but one week after the second vaccination in mice without preimmunization. Low ELISA antibody levels against YFV were observed in mice preimmunized with one human dose, probably because of low sensitivity in the ELISA system where rabbit anti-DENV2 antibody was used to cross-reactively capture YFV antigen. However, the levels were significantly higher than those obtained with non-preimmunized mice following the first vaccination. These results indicated that antibody titers/levels induced by the dengue tetravalent DNA vaccine were not affected by preimmunization with YFV in mice, except for slight and sporadic increases against certain types of dengue viruses.

\section{Pre- and postvaccination IgG isotype profiles}

Antibodies induced by the dengue tetravalent DNA vaccine in mice preimmunized with flavivirus antigens were further analyzed to determine their $\operatorname{IgG} 1$ and $\operatorname{IgG} 2 \mathrm{a}$ isotype profile to each immunogen. DNA vaccines are known to induce Th1-type immune responses with an isotype profile of $\lg G 2 \mathrm{a}>\lg G 1$ in mice, when inoculated by the intramuscular route [5].As shown in (Table 2), mice vaccinated with the dengue tetravalent DNA vaccine by needle-free jet injection into the thigh (without preimmunization) also developed higher $\lg G 2 a$ than IgG1 antibody titers against all four types of dengue virus. Preimmunization with DENV2 induced IgG2a antibody titers higher than the IgG1 antibody titers against DENV1-3. After vaccination, the 
Citation: Konishi E, Takizawa Y (2010) Effect of Pre-Existing Immunity to Flaviviruses on Balanced Induction of Neutralizing Antibodies by a Dengue Tetravalent DNA Vaccine in Mice. J Vaccines Vaccin 1: 102. doi:10.4172/2157-7560.1000102

Page 5 of 7

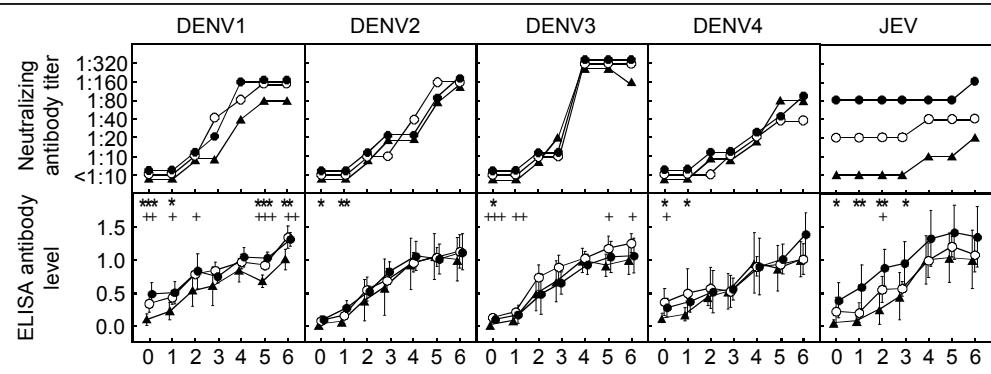

Weeks after the first vaccination

Figure 3: Effect of preimmunity to JEVAX on immunogenicity of the dengue tetravalent DNA vaccine. Groups of six mice were preimmunized by two inoculations, with a 2-week interval, with a 1/2 (closed circle) or 1/10 (open circle) human dose of JEVAX or inoculated with PBS (closed triangle). Two and six weeks after the second inoculation for preimmunization, these mice were vaccinated with $40 \mu \mathrm{g}$ of the dengue tetravalent DNA vaccine (10 $\mu \mathrm{g}$ for each type). Pooled sera were examined for neutralizing antibody titers (upper panel) and individual sera were examined for ELISA antibody levels (lower panel) against DENV1-4 and JEV (indicated above the upper panel). For ELISA, mean ELISA values were plotted with SD (indicated by bars). Significant differences in mean ELISA values from non-preimmunized mice were indicated by * $(P<0.05)$, ${ }^{* *}(P<0.01)$ and ${ }^{* *}(P<0.001)$ for mice preimmunized with a $1 / 2$ dose of JEVAX and $+(P<0.05),++(P<0.01)$ and $+++(P<0.001)$ for mice preimmunized with a $1 / 10$ dose of JEVAX.

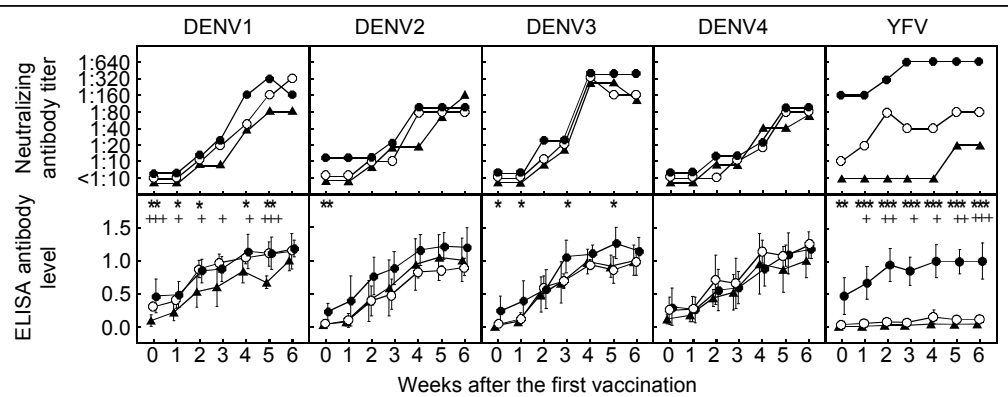

Figure 4: Effect of preimmunity to the 17D strain of YFV on immunogenicity of the dengue tetravalent DNA vaccine. Groups of six mice were preimmunized by two inoculations, with a two-week interval, with $5.6 \times 10^{6}$ (closed circle) or $5.6 \times 10^{4}$ (open circle) PFU of YFV or inoculated with PBS (closed triangle). Two and six weeks after the second inoculation for preimmunization, these mice were vaccinated with $40 \mu \mathrm{g}$ of the dengue tetravalent DNA vaccine (10 $\mu \mathrm{g}$ for each type). Pooled sera were examined for neutralizing antibody titers (upper panel) and individual sera were examined for ELISA antibody levels (lower panel) against DENV1-4 and YFV (indicated above the upper panel). The positive control for the DENV1-4 ELISA was obtained from mice only vaccinated with the tetravalent vaccine, while that for the YFV ELISA was obtained from mice preimmunized with $5.6 \times 10^{6}$ PFU of YFV and then vaccinated with the tetravalent vaccine. Since this experiment was performed in parallel to the experiment shown in Figure 3, time courses obtained with the control mice (closed triangles) are the same as those shown in Figure 3. Significant differences in mean ELISA values from non-preimmunized mice were indicated by * $(P<0.05),{ }^{* *}(P<0.01)$ and ${ }^{* *}(P<0.001)$ for mice preimmunized with a $1 / 2$ dose of JEVAX and + $(P<0.05),++(P<0.01)$ and $+++(P<0.001)$ for mice preimmunized with a $1 / 10$ dose of JEVAX.

\begin{tabular}{|c|c|c|c|c|c|c|c|}
\hline \multirow{2}{*}{ Preimmunized with: } & \multirow[b]{2}{*}{ Week $^{\mathrm{b}}$} & \multicolumn{6}{|c|}{$\operatorname{lgG} 2 \mathrm{a} / \lg \mathrm{g} 1$ ratio $^{\mathrm{c}}$} \\
\hline & & DENV1 & DENV2 & DENV3 & DENV4 & JEV & YFV \\
\hline \multirow[t]{2}{*}{ DENV2 } & 0 & 64 & 2 & 64 & 1 & $-d$ & - \\
\hline & 6 & 2048 & 16 & 512 & 8 & - & - \\
\hline Control $^{a}$ & 6 & 512 & 16 & 64 & 16 & - & - \\
\hline \multirow[t]{2}{*}{ JEVAX } & 0 & $1 / 2$ & $1 / 4$ & $1 / 64$ & $1 / 2$ & 1 & - \\
\hline & 6 & 16 & 2 & 8 & 2 & 1 & - \\
\hline Control & 6 & 512 & 32 & 16 & 16 & 8 & - \\
\hline \multirow[t]{2}{*}{ YFV } & 0 & 4 & $1 / 8$ & 1 & $1 / 4$ & - & 4 \\
\hline & 6 & 64 & 1 & 4 & 4 & - & 1 \\
\hline Control & 6 & 512 & 32 & 16 & 16 & - & 8 \\
\hline
\end{tabular}

alnoculated with PBS.

'Period (week) after the first vaccination with the dengue tetravalent DNA vaccine. "0" indicates the time point just before the first vaccination.

'The ratio of IgG2a to IgG1 antibody titers against each of the viruses: DENV1-4, JEV and YFV.

'Not tested.

Table 2: IgG isotype profiles of the antibodies induced in mice preimmunized with flavivirus antigens and then vaccinated with the dengue tetravalent DNA vaccine.

$\operatorname{IgG2a} / \operatorname{lgG} 1$ ratio increased for all types of dengue viruses. On the other hand, preimmunization with JEVAX induced higher $\operatorname{lgG} 1$ than IgG2a antibody titers (with $\operatorname{IgG} 2 \mathrm{a} / \operatorname{lgG} 1$ ratios of less than one) for DENV1-4. However, the IgG2a/lgG1 ratio increased to greater than one following vaccination. Similarly, mice preimmunized with YFV showed increased $\operatorname{lgG} 2 \mathrm{a} / \operatorname{lgG} 1$ ratios for all types of dengue viruses after vaccination. Antibodies against DENV1 showed the highest IgG2a/ $\lg G 1$ ratio, irrespective of whether or not mice were preimmunized and irrespective of the immunogen used for preimmunization. In mice preimmunized with JEVAX or YFV, vaccination with the dengue DNA vaccine did not increase the $\operatorname{lgG} 2 \mathrm{a} / \operatorname{lgG} 1$ ratio for antibodies to JEV or YFV. These results indicated that the dengue tetravalent DNA vaccine 
induced higher IgG2a than $\operatorname{lgG} 1$ antibody titers against DENV1-4 even in preimmunized mice, as far as preimmunization with DENV2, JEVAX or YFV was concerned. Attempts to detect any relationship between the $\lg G 2 \mathrm{a} / \operatorname{lgG} 1$ antibody ratio and the neutralizing antibody titers failed, probably because the neutralizing antibody titers of sera with an isotype profile of $\lg G 1>\lg G 2$ a were mostly $<1: 10$.

\section{Discussion}

This study assessed the effects of preimmunity to DENV2, JEVAX and YFV on immunogenicity of the dengue tetravalent DNA vaccine in mice. Most members of the genus Flavivirus are grouped into eight antigenic complexes, within which members show high serological cross-reactivities [7]. DENV1-4 constitute the dengue virus serocomplex, while JEV and YFV belong to other serocomplexes. When preimmunized with DENV2, mice developed neutralizing antibodies only against DENV2 as expected, this implied imbalanced antibody responses against DENV1-4 before vaccination with the tetravalent vaccine. However, after two vaccinations, mice developed neutralizing antibody titers efficiently against other dengue types and the titers against DENV1-4 at the end of the experimental period ( 6 weeks after the first vaccination) were 4 - to 8 -fold higher than those induced in vaccinated mice without preimmunization, providing balanced neutralizing antibody responses against DENV14. Preimmunization with viral antigens of other serocomplexes (JEV and YFV) sporadically increased neutralizing antibody titers against certain types of viruses (maximum of four-fold), but did not significantly affect the ability of the dengue tetravalent DNA vaccine to induce balanced antibody responses. Although pooled sera were used for neutralization tests in the present study, individual variations in antibody titers were usually within 2-fold in mice immunized with dengue or JE DNA vaccines in our previous studies [16,21].

Neutralizing antibody titers induced by the dengue tetravalent DNA vaccine were increased by preimmunization with only one type of dengue virus (DENV2) not only against the homotype but also against the viral heterotypes. Increases in the antibody titer against heterologous viruses are partly explained by crossneutralization. However, the increase was larger than that estimated by cross-neutralization. For instance, the neutralizing antibody titer against DENV1 at week 6 of vaccination was $1: 80$ in mice without preimmunization and 1:320 in mice preimmunized with $5.0 \times 10^{7} \mathrm{PFU}$ of DENV2 antigen. Since hyperimmune sera against DENV2 showed a neutralizing antibody titer of 1:2560 against DENV2 and 1:10 against DENV1 (cross-neutralization titer of $1 / 256$; Figure 1), it is expected that serum showing an antibody titer of 1:1280 against DENV2 only contributed towards increasing the titer to 1:5 against DENV1. Thus, the difference between the 1:80 and 1:320 antibody titers cannot be explained solely by cross-reactivity between DENV1 and DENV2 in our neutralization test. A potential explanation for this observation is an enhancement in the immunogenicity of pcD1ME, a DENV1 antigen component of the dengue tetravalent DNA vaccine, in cases where mice had previously exhibited immune responses to DENV2, which resulted in induction of broad cross-reactive neutralizing antibodies.

The enhanced induction of cross-neutralizing antibodies shown in this study was demonstrated by using a dengue tetravalent DNA vaccine and an additional antigen prior to vaccination. A similar phenomenon was previously shown in a mouse model using our dengue tetravalent DNA vaccine, but the additional antigen was used after vaccination [20]. Specifically, groups of mice, which had been vaccinated twice with the dengue tetravalent DNA vaccine, were inoculated with each of the DENV1-4 antigens in the form of infected mouse brain homogenates 3 weeks after the second immunization. The heterotypic, as well as the homotypic, neutralizing antibody titers were significantly elevated after the inoculation. For instance, mice vaccinated with the tetravalent DNA vaccine and then inoculated with DENV2 developed neutralizing antibody titers of 1:640 against DENV2 and 1:320 against DENV1, whereas the control mice vaccinated only with a DENV2 DNA vaccine and then inoculated with DENV2 developed titers of 1:1280 against DENV2 and as little as 1:40 against DENV1. The high-level induction of cross-neutralizing antibodies has also been reported previously in mice co-immunized with the dengue tetravalent DNA vaccine and a protein vaccine that consisted of DENV2 subviral extracellular particles [16]. The neutralizing antibody titer against DENV1 induced by the tetravalent DNA vaccine alone (1:20) was increased to $1: 160$ by co-immunization with the DENV2 protein vaccine. This 8 -fold increase was much higher than expected from the cross-reactivity of the DENV2 antibody induced by the DENV2 protein vaccine alone (neutralizing antibody titer of $<1: 10$ against DENV1).

Immune responses against sequential exposures to dengue and other flavivirus antigens in humans have been intensively studied using live attenuated dengue and YF vaccines $[1,17]$. As described above, DENV1-4 and YFV belong to different serocomplexes: thus, serological cross-reactivities between DENV1-4 and YFV are less than those among DENV1-4. Although the present study only showed a slight effect of preimmunization with YFV on immunogenicity of the dengue tetravalent DNA vaccine in mice, clinical trials evaluating live attenuated dengue vaccine candidates suggest that preimmunity to YFV can increase immune responses against DENV on subsequent dengue vaccination. Specifically, a live attenuated DENV2 vaccine showed a significantly higher seroconversion rate in YFV-immune than non-immune recipients [90\% vs. $61 \%$; $\mathrm{P}<0.01$ : 1]. Moreover, monovalent live attenuated vaccine against each of the DENV1-4 induced neutralizing antibody responses against all four types of dengue virus in volunteers with immunologic memory against YFV, while only monovalent antibody responses were shown in most naïve volunteers [17].

It is generally accepted that almost all individuals who survive infection with two different types of dengue virus are then protected from infection by all four virus types [7]. Considering the situation in endemic areas, the enhanced cross-neutralizing antibody responses shown in this study potentially contribute to long-term immune responses against all types after dengue tetravalent vaccines. It is likely that individuals who receive the dengue tetravalent DNA vaccine develop balanced neutralizing antibody responses against all four types of dengue virus irrespective of whether they acquire infection with a single type before and after vaccination. The observation in the present study may be consistent with a complementary mechanism suggested by dengue chimeric vaccine studies using heterologous prime-boost immunizations $[8,9]$. It is speculated that this phenomenon is related to the enhanced neutralizing antibody responses against cross-reactive antigenic epitope(s), possibly by a mechanism involving molecular mimicry of an epitope presented on the envelope proteins of DENV1-4 that is recognized by immune cells.

In conclusion, preimmunization with DENV2, JEVAX or YFV did not affect the ability of our dengue tetravalent DNA vaccine to induce balanced neutralizing antibody responses in a mouse model. In endemic areas where people are frequently exposed to dengue virus infection, the tetravalent vaccine is considered to induce relatively strong cross-reactive neutralizing antibodies, which is important for durable vaccine effectiveness against all four types of dengue virus. 
Citation: Konishi E, Takizawa Y (2010) Effect of Pre-Existing Immunity to Flaviviruses on Balanced Induction of Neutralizing Antibodies by a Dengue Tetravalent DNA Vaccine in Mice. J Vaccines Vaccin 1: 102.doi:10.4172/2157-7560.1000102

Page 7 of 7

\section{Acknowledgments}

This study was supported in part by grants from the Research on Emerging and Re-emerging Infectious Diseases of the Ministry of Health, Labour and Welfare of Japan (H21-shinkou-ippan-005) and the Science and Technology Research Partnership for Sustainable Development (SATREPS) of the Japan Science and Technology Agency/Japan International Cooperation Agency (JST/JICA).

\section{References}

1. Bancroft WH, Scott RM, Eckels KH, Hoke CH Jr, Simms TE et al. (1984) Dengue virus type 2 vaccine: reactogenicity and immunogenicity in soldiers. $J$ Infect Dis 149: 1005-1010.

2. Barnett ED, Kozarsky PE, Steffen R (2008) Vaccines for International travel. In: Plotkin SA, Orenstein WA, Offit PA (eds) Vaccines 5th edition, Saunders Elsevier, Maryland Heights, pp 1431-52.

3. Blaney JE Jr, Durbin AP, Murphy BR, Whitehead SS (2006) Development of a live attenuated dengue virus vaccine using reverse genetics. Viral Immunol 19: $10-32$.

4. Burke DS, Nisalak A, Johnson DE, Scott RM (1988) A prospective study of dengue infections in Bangkok. Am J Trop Med Hyg 38: 172-180

5. Feltquate DM, Heaney S, Webster RG, Robinson HL (1997) Different T helper cell types and antibody isotypes generated by saline and gene gun DNA immunization. J Immunol 158: 2278-2284

6. Green S, Rothman A (2006) Immunopathological mechanisms in dengue and dengue hemorrhagic fever. Curr Opin Infect Dis 19: 429-436.

7. Gubler DJ, Kuno G, Markoff L (2007) Flaviviruses. In: Knipe DM, Howley PM (eds) Fields virology 5th edition, Lippincott Williams \& Willkins, Philadelphia, pp 1153-252.

8. Guirakhoo F, Kitchener S, Morrison D, Forrat R, McCarthy K et al. (2006) Live attenuated chimeric yellow fever dengue type 2 (ChimeriVax-DEN2) vaccine: Phase I clinical trial for safety and immunogenicity: effect of yellow fever preimmunity in induction of cross neutralizing antibody responses to all 4 dengue serotypes. Hum Vaccin 2: 60-67.

9. Guy B, Barban V, Mantel N, Aguirre M, Gulia S et al (2009) Evaluation of interferences between dengue vaccine serotypes in a monkey model. Am J Trop Med Hyg 80: 302-311.

10. Guy B, Guirakhoo F, Barban V, Higgs S, Monath TP, et al. (2010) Preclinical and clinical development of YFV 17D-based chimeric vaccines against dengue, West Nile and Japanese encephalitis viruses. Vaccine 28: 632-649.

11. Guzman MG, Kouri GP, Bravo J, Soler M, Vazquez S, et al. (1990) Dengue hemorrhagic fever in Cuba, 1981: a retrospective seroepidemiologic study. Am J Trop Med Hyg 42: 179-184.

12. Halstead SB (2007) Dengue. Lancet 370(9599): 1644-1652.

13. Halstead SB (2003) Neutralization and antibody-dependent enhancement of dengue viruses. Adv Virus Res 60:421-467.

14. Halstead SB, Vaughn DW (2008) Dengue vaccines. In: Plotkin SA, Orenstein WA, Offit PA (eds) Vaccines 5th edition, Saunders Elsevier, Maryland Heights, pp 1155-61.

15. Hombach J, Cardosa ML, Sabchareon A, Vaughn DW, Barrett AD (2007) Scientific consultation on immunological correlates of protection induced by dengue vaccines report from a meeting held at the World Health Organization 17-18 November 2005. Vaccine 25: 4130-4139.

16. Imoto J, Konishi E (2007) Dengue tetravalent DNA vaccine increases its immunogenicity in mice when mixed with a dengue type 2 subunit vaccine or an inactivated Japanese encephalitis vaccine. Vaccine 25: 1076-1084.
17. Kanesa-Thasan N, Sun W, Ludwig GV, Rossi C, Putnak JR et al. (2003) Atypical antibody responses in dengue vaccine recipients. Am J Trop Med Hyg 69: 32-38.

18. Kitchener S, Nissen M, Nasveld P, Forrat R, Yoksan S et al. (2006) Immunogenicity and safety of two live-attenuated tetravalent dengue vaccine formulations in healthy Australian adults. Vaccine 24:1238-1241.

19. Kliks SC, Nisalak A, Brandt WE, Wahl L, Burke DS (1989) Antibody-dependent enhancement of dengue virus growth in human monocytes as a risk factor for dengue hemorrhagic fever. Am J Trop Med Hyg. 40: 444-451.

20. Konishi E, Kosugi S, Imoto J (2006) Dengue tetravalent DNA vaccine inducing neutralizing antibody and anamnestic responses to four serotypes in mice. Vaccine 24: 2200-2207.

21. Konishi E, Terazawa A, Imoto J (2003) Simultaneous immunization with DNA and protein vaccines against Japanese encephalitis or dengue synergistically increases their own abilities to induce neutralizing antibody in mice. Vaccine 21: $1826-32$

22. Konishi E, Yagawa K, Yamanaka A (2008) Vero cells infected with vaccinia viruses expressing Japanese encephalitis virus envelope protein induce polykaryocyte formation under neutral conditions. Jpn J Infect Dis 61: 410-411.

23. Kuno G (2003) Serodiagnosis of flaviviral infections and vaccinations in humans. Adv Virus Res. 61: 3-65.

24. Kurane I, Mady BJ, Ennis FA (1991) Antibody-dependent enhancement of dengue virus infection. Rev Med Virol 1: 211-221.

25. Lindenbach BD, Thiel HJ, Rice CM (2007) Flaviviridae: the viruses and their replication. In: Knipe DM, and Howley PM (eds) Fields virology 5th edition, Lippincott Williams \& Willkins, Philadelphia , pp 1101-52.

26. Lu S, Wang S, Grimes-Serrano JM (2008) Current progress of DNA vaccine studies in humans. Expert Rev Vaccines 7: 175-191.

27. Monath TP, Cetron MS, Teuwen DE (2008) Yellow fever vaccine. In: Plotkin SA Orenstein WA, Offit PA (eds) Vaccines 5th edition, Saunders Elsevier, Maryland Heights, pp 959-1055.

28. Morrison D, Legg TJ, Billings CW, Forrat R, Yoksan S, et al. (2010) A nove tetravalent dengue vaccine is well tolerated and immunogenic against all 4 serotypes in flavivirus-naive adults. J Infect Dis 201: 370-307.

29. Sabchareon A, Lang J, Chanthavanich $P$, Yoksan S, Forrat $P$, Attanath $P$ et al. (2002) Safety and immunogenicity of tetravalent live-attenuated dengue vaccines in Thai adult volunteers: role of serotype concentration, ratio, and multiple doses. Am J Trop Med Hyg 66: 264-272.

30. Sabin AB (1952) Research on Dengue during World War II. Am J Trop Med Hyg 1: 30-50.

31. Stephenson JR (2005) Understanding dengue pathogenesis: implications for vaccine design. Bull World Health Organ 83: 308-314.

32. Sun W, Cunningham D, Wasserman SS, Perry J, Putnak JR, et al. (2009) Phase 2 clinical trial of three formulations of tetravalent live-attenuated dengue vaccine in flavivirus-naïve adults. Hum Vaccin 5: 33-40.

33. Whitehead SS, Durbin AP (2010) Prospects and challenges for dengue virus vaccine development. In: Hanley KA, Weaver SC (eds) Frontiers in Dengue Virus Research. Caister Academic Press, Norfolk UK, pp 221-237.

34. Wright PF, Durbin AP, Whitehead SS, Ikizler MR, Henderson S, Blaney JE, Thumar B, Ankrah S, Rock MT, McKinney BA, MurphyBR, Schmidt AC (2009) Phase 1 trial of the dengue virus type 4 vaccine candidate rDEN4\{Delta\}30-4995 in healthy adult volunteers. Am J Trop Med Hyg 81: 834-841.

35. Yamanaka A, Kosugi S, Konishi E (2008) Infection-enhancing and -neutralizing activities of mouse monoclonal antibodies against dengue type 2 and 4 viruses are controlled by complement levels. J Virol 82: 927-37. 